\title{
Secure Smart Charger: Plataforma de segurança cibernética para sensoriamento de corrente elétrica
}

\author{
João P. D. Antiquera, Francisco A. S. do Carmo, Guilherme P. Aquino e Evandro C. Vilas Boas
}

\begin{abstract}
Resumo-Esse trabalho descreve o projeto de uma plataforma de segurança cibernética para sensoriamento de corrente elétrica em carregadores de carros elétricos. $O$ sistema integra um aplicativo móvel, um banco de dados e um módulo de hardware acoplado em um carregador de carro elétrico convencional para mensurar a potência transferida para o veículo. Emprega-se o protocolo MQTT (Message Queuing Telemetry Transport) para fins de comunicação. Implementa-se o protocolo TLS (Transport Layer Security) para prover comunicação segura fim-a-fim.
\end{abstract}

\section{Palavras-Chave-IoT, MQTT, segurança cibernética.}

Abstract - This work presents the development of noninvasive electric current sensing cybersecurity platform for electric vehicle chargers. The system comprises a mobile application, database, and hardware module coupled to a conventional electric vehicle charger, which measures the power transferred to the vehicle. The Message Queuing Telemetry Transport (MQTT) protocol provides communication between the system elements. The Secure Sockets Layer (SSL) protocol establishes secure end-to-end communication.

Keywords - Cyber security, IoT, MQTT.

\section{INTRODUÇÃO}

A conexão de sensores e atuadores em uma rede de computador ou à Internet refere-se aos conceitos básicos que definem a Internet das Coisas (IoT, Internet of Things) [1]. Os sensores obtêm informações diversas. Processam-se esses dados em diferentes pontos da rede e, em alguns casos, definem-se intervenções no ambiente por meio de atuadores. Dentre as diversas aplicações IoT, destacam-se soluções para o setor automobilístico no desenvolvimento de sistemas de recarga inteligentes para carros elétricos [2].

O Inatel (Instituto Nacional de Telecomunicações) foi contemplado com um carregador de carros elétricos, que será instalado no campus para uso comunitário. Esse carregador não dispõe de um sistema de gerência e monetização do consumo de energia elétrica. Portanto, desenvolveu-se um sistema de sensoriamento de corrente elétrica não invasivo para implementar essas funcionalidades [3]. Na primeira versão, restringiu-se a comunicação do sistema ao uso de rede cabeada Ethernet e não se aplicaram aspectos relacionados à proteção de dados. Portanto, esse trabalho explorou autenticação e criptografia para implementar segurança cibernética à primeira versão. Além disso, desenvolveu-se um aplicativo móvel para interação do usuário com o sistema, implementou-se um broker MQTT (Message Queuing Telemetry Transport) próprio para o projeto e substitui-se a conexão à Internet cabeada pela sem fio via rede $\mathrm{WiFi}$.

João P. D. Antiquera, Francisco A. S. do Carmo, Guilherme P. Aquino e Evandro C. V. Boas, Laboratório de Cyber Segurança e Internet das Coisas (CS\&I Lab.) Instituto Nacional de Telecomunicações (Inatel) joao_antiquera@get.inatel, francisco.assis@inatel.br, guilhermeaquino@inatel.br, evandro.cesar@inatel.br.

\section{PlATAFORMA DE SEGURANÇA CIBERNÉTICA PARA \\ SENSORIAMENTO DE CORRENTE ELÉTRICA NÃO INVASIVO}

O sistema de sensoriamento de corrente elétrica não invasivo emprega um sensor de corrente acoplado ao cabo do carregador para leitura do fluxo de corrente elétrica. O microcontrolador ESP8266 trata o sinal de corrente proveniente do sensor e determina a potência em Watts (W), a corrente elétrica em Ampére (A) e o valor da recarga em reais ( $\mathrm{R} \$)$. Enviam-se esses dados para armazenamento em um banco de dados, disponibilizando-os para consulta do usuário por meio do aplicativo móvel. Estabelece-se a comunicação entre o microcontrolador, o banco de dados e o aplicativo móvel por meio de um servidor broker MQTT.

Para adaptar o carregador de carro elétrico de maneira não invasiva, optou-se pelo uso do sensor de corrente YHDC SCT013-100 [4]. Esse sensor mensura correntes elétricas alternadas de entrada entre 0 e $100 \mathrm{~A}$, fornecendo corrente alternada de saída entre 0 e $50 \mathrm{~mA}$. Implementou-se o circuito, visto na Figura 1, para manipular a corrente de saída do sensor de modo a obter um sinal de tensão proporcional, adaptando-o para a leitura pelo microcontrolador ESP8266. Utilizou-se um circuito de offset composto por um divisor de tensão com resistores de igual valor $(10 \mathrm{k} \Omega)$ e um capacitor eletrolítico $(100 \mathrm{uF})$ em paralelo a um dos resistores. Empregou-se um resistor de carga para gerar um sinal de tensão de saída alternado com nível médio igual a metade do valor da tensão de alimentação do circuito divisor de tensão.

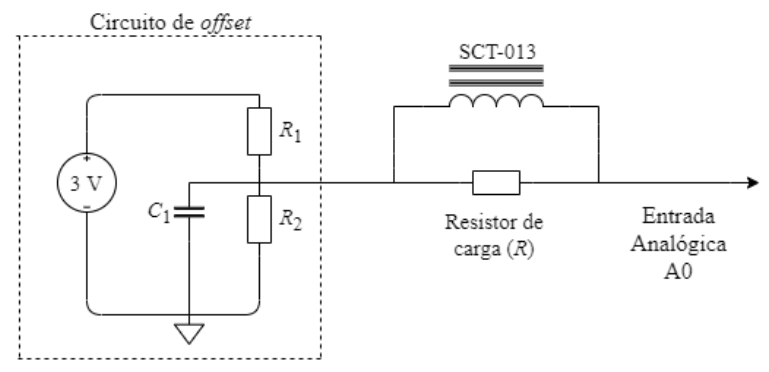

Fig. 1. Circuito para adaptação do sinal de corrente do sensor ao NodeMCU.

Calibrou-se a resistência do resistor de carga $(R)$ para a tensão de referência do microcontrolador $\left(V_{m}\right)$, o número de espiras da bobina do sensor $(N=2000)$ e a corrente eficaz máxima do condutor a ser medido $\left(I_{\mathrm{RMS}}\right)$. A relação entre esses parâmetros é dada por:

$$
R(\Omega)=\frac{\left(V_{m} \times N\right)}{\left(2 \sqrt{2} \times I_{R M S}\right)} .
$$

Na Figura 2, visualizam-se os componentes de hardware do projeto. O microcontrolador trata o sinal de tensão de saída por meio de funções da biblioteca EmonLib.h. Inicialmente, coletase o sinal de tensão por meio do pino analógico e obtém-se a corrente eficaz. Utiliza-se essa corrente para cálculo da potência 
consumida. Posteriormente, monetiza-se o consumo pela relação:

$$
C(R \$)=\int P(t) d t \times K
$$

onde $C$ é o consumo em reais, $P(t)$ é a potência variante no tempo em Watts e $K$ a constante que representa o valor do $\mathrm{KW} / h$ da concessionária de energia.

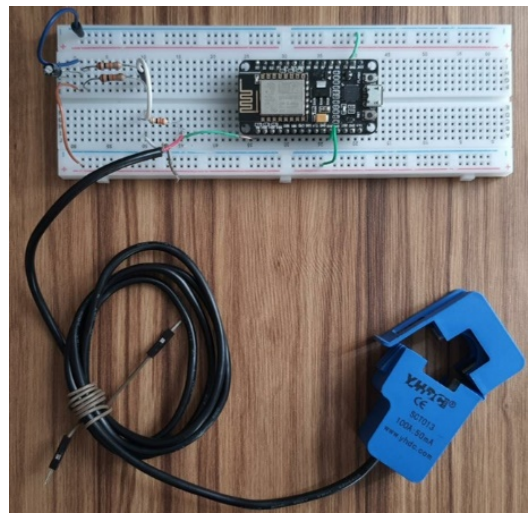

Fig. 2. Componentes de hardware do sistema de sensoriamento.

Desenvolveram-se bancos de dados para registros de usuários e histórico de recargas, assim como um aplicativo móvel para interação entre o usuário e o sistema. Implementouse o banco de dados por meio da plataforma de código aberto LAMP (Linux, Apache, MySQL e PHP). Utilizou-se a linguagem Java e a plataforma Android Studio para desenvolver o aplicativo móvel. Na Figura 3, visualizam-se as telas do aplicativo, que compreende as funções de cadastro, login, recarga e histórico de recargas.

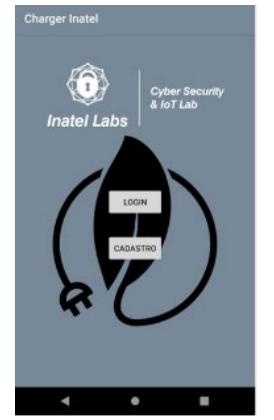

Tela de acesso/registro

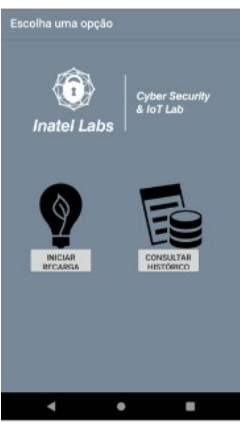

Funcionalidades do App

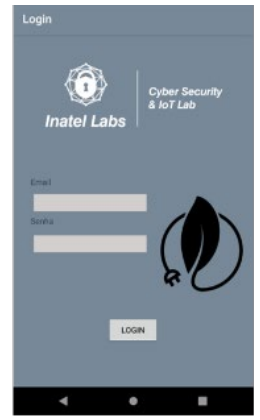

Tela de login

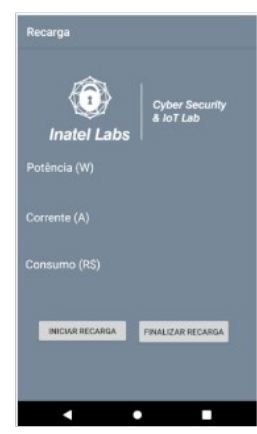

Iniciar/finalizar recarga

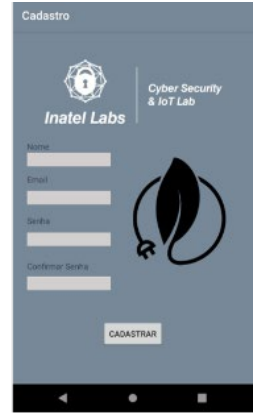

Tela de cadastro

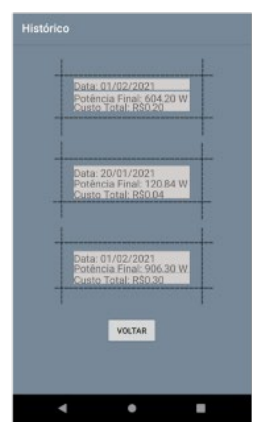

Histórico de recargas
Fig. 3. Aplicativo móvel.

\section{APLICAÇ̃̃O DO PROTOCOLO TLS}

Para implementar a comunicação segura fim-a-fim, utilizouse o protocolo TLS (Transport Layer Security) na implementação do aplicativo móvel, banco de dados e módulo de hardware. Para o aplicativo, empregaram-se as bibliotecas do pacote Paho Java MQTT Client. Acrescentou-se a conexão MQTT em um Android-Service, cuja a execução ocorre em back-end e mantém a conexão ativa enquanto o aplicativo alterna entre as diversas atividades. Em hardware, utilizou-se um arquivo de controle ("secret.h") e um certificado TLS ("server.crt"). Embarcaram-se os arquivos no microcontrolador por meio da codificação. Para estabelecer uma comunicação segura, leem-se e validam-se esses arquivos inicialmente para estabelecer as demais comunicações de maneira criptografada. $\mathrm{Na}$ Figura 4, demonstram-se o processo de criptografia implementado por meio de capturas de quadros pela ferramenta Wireshark, garantindo a troca de dados segura pela plataforma.

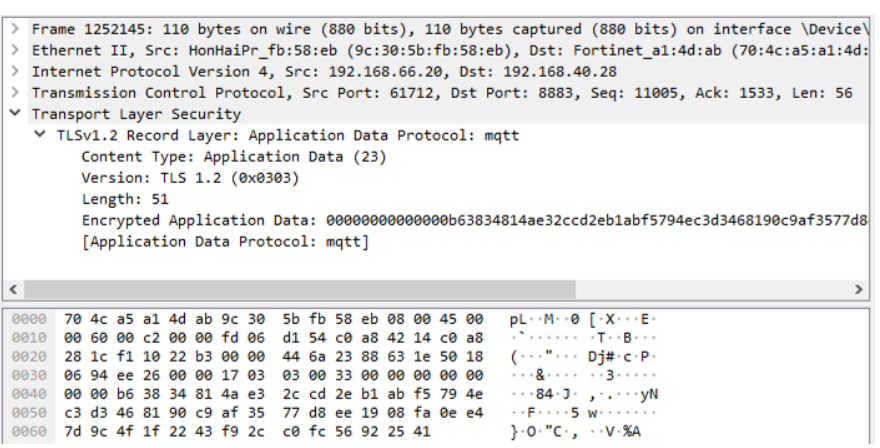

Fig. 4. Captura da troca de mensagens entre as entidades da plataforma.

\section{CONCLUSÕES}

Esse trabalho abordou o desenvolvimento de uma plataforma de segurança cibernética para sensoriamento de corrente elétrica para carregadores de carro elétrico. Empregou-se um sensor de corrente não invasivo para aferir o consumo de potência de um determinado usuário durante uma recarga, monetizando-a. Desenvolveram-se bancos de dados para armazenar os dados de recargas, disponibilizando essas informações para consulta por meio de um aplicativo. Implementou-se o protocolo TLS para prover comunicação segura fim-a-fim. Obtiveram-se resultados satisfatórios durante os testes. Identificou-se a possibilidade de mensurar diferentes faixas de corrente elétrica, permitindo expandir as aplicações do projeto para mensurar o consumo de outros tipos de carga.

\section{AGRADECIMENTOS}

Os autores agradecem ao Instituto Nacional de Telecomunicações - Inatel, por prover os meios necessários a realização desse trabalho de IC.

\section{REFERÊNCIAS}

[1] A. Al-Fuqaha et al., "Internet of Things: A Survey on Enabling Technologies, Protocols, and Applications," in IEEE Communications Surveys \& Tutorials, vol. 17, no. 4, pp. 2347-2376, 2015.

[2] Computer World, BMW lança rede inteligente de carregadores para carros elétricos híbridos. [Online]. Disponível em: <https://computer world.com.br/inovacao/bmw-lanca-rede-inteligente-de-carregadores-par a-carros-eletricos-e-hibridos/>. Acesso: 2020-08-31.

[3] Italo A. S. et al., "Sistema de sensoriamento de corrente elétrica utilizando IoT," in XXXII Congresso de Iniciação Científica do Inatel Incitel 2020, Santa Rita do Sapucaí, MG, Brasil: Instituto Nacional de Telecomunicacões, 2020, pp. 54-57. ISBN: 2359-6457.

[4] YHDC.Splitcorecurrenttransformer. [Online]. Disponível em: $<$ https://im g.filipeflop.com/files/download/DatasheetSCT013.pdf> . Acesso: 202008-31. 